\title{
Hydrophobically-associating cationic polymers as micro-bubble surface modifiers in dissolved air flotation for cyanobacteria cell separation
}

\author{
R.K.L. Yap ${ }^{a, b}$, M. Whittaker ${ }^{c}$, M. Diao ${ }^{d}$, R.M. Stuetz $^{a}$, B. Jefferson ${ }^{e}$, \\ V. Bulmus ${ }^{f}$, W.L. Peirson ${ }^{g}$, A.V. Nguyen ${ }^{d}$, R.K. Henderson ${ }^{a, 1, *}$ \\ ${ }^{a}$ UNSW Water Research Centre, School of Civil and Environmental Engineering, The University of New South Wales, \\ Sydney, NSW 2052, Australia \\ ${ }^{\mathrm{b}}$ Centre for Advanced Macromolecular Design, School of Chemical Engineering, The University of New South Wales, \\ Sydney, NSW 2052, Australia \\ c Monash Institute of Pharmaceutical Sciences (MIPS), Monash University, IVC 3052, Australia \\ d School of Chemical Engineering, The University of Queensland, Brisbane, Queensland 4072, Australia \\ e Cranfield Water Science Institute, School of Applied Sciences, Cranfield University, Bedfordshire MK43 OAL, UK \\ ${ }^{\mathrm{f}}$ Department of Chemical Engineering, Izmir Institute of Technology, Urla, 35430 Izmir, Turkey \\ ${ }^{\mathrm{g}}$ Water Research Laboratory, School of Civil and Environmental Engineering, The University of New South Wales, \\ Manly Vale, NSW 2093, Australia
}

\section{A R T I C L E I N F O}

Article history:

Received 17 February 2014

Received in revised form

15 May 2014

Accepted 18 May 2014

Available online 2 June 2014

Keywords:

Algae separation

Cationic bubbles

Cyanobacteria

Flotation

PosidAF

Water soluble polymers

\begin{abstract}
A B S T R A C T
Dissolved air flotation (DAF), an effective treatment method for clarifying algae/cyanobacteria-laden water, is highly dependent on coagulation-flocculation. Treatment of algae can be problematic due to unpredictable coagulant demand during blooms. To eliminate the need for coagulation-flocculation, the use of commercial polymers or surfactants to alter bubble charge in DAF has shown potential, termed the PosiDAF process. When using surfactants, poor removal was obtained but good bubble adherence was observed. Conversely, when using polymers, effective cell removal was obtained, attributed to polymer bridging, but polymers did not adhere well to the bubble surface, resulting in a cationic clarified effluent that was indicative of high polymer concentrations. In order to combine the attributes of both polymers (bridging ability) and surfactants (hydrophobicity), in this study, a commercially-available cationic polymer, poly(dimethylaminoethyl methacrylate) (polyDMAEMA), was functionalised with hydrophobic pendant groups of various carbon chain lengths to improve adherence of polymer to a bubble surface. Its performance in PosiDAF was contrasted against commercially-available poly(diallyl dimethyl ammonium chloride) (polyDADMAC). All synthesised polymers used for bubble surface modification were found to produce positively charged bubbles. When applying these cationic micro-bubbles in PosiDAF, in the absence of coagulation-flocculation, cell removals in excess of $90 \%$ were obtained, reaching a maximum of $99 \%$ cell removal and thus demonstrating process viability. Of the synthesised polymers, the polymer
\end{abstract}

\footnotetext{
* Corresponding author.

E-mail address: r.henderson@unsw.edu.au (R.K. Henderson).

${ }^{1}$ Current address: School of Chemical Engineering, The University of New South Wales, Sydney, NSW 2052, Australia. http://dx.doi.org/10.1016/j.watres.2014.05.032

0043-1354/@ 2014 Elsevier Ltd. All rights reserved.
} 
containing the largest hydrophobic functionality resulted in highly anionic treated effluent, suggesting stronger adherence of polymers to bubble surfaces and reduced residual polymer concentrations.

() 2014 Elsevier Ltd. All rights reserved.

\section{Introduction}

Dissolved air flotation (DAF) is a solid-liquid separation process in which nucleated microbubbles are introduced to a suspension comprising flocculated particles. Collision and attachment of bubbles and particles create low density bubble-particle agglomerates which rise to the surface to form a float layer and can then be removed mechanically or hydraulically. In water and wastewater treatment plants (WTPs/ WWTPs), DAF is used for the removal of low density contaminants such as algae and natural organic matter (NOM) from reservoir water or waste stabilisation ponds (WSPs). Coagulation-flocculation is conventionally applied to reduce particle and colloid charge, increase particle sizes, complex with NOM and ensure bubble-particle interactions and subsequent removal efficiencies are optimal (Edzwald, 2010).

The presence of algae and cyanobacteria in raw water can present a significant challenge for WTP/WWTP operators and DAF is becoming a popular process option to improve treatability (Edzwald, 2010; Teixeira and Rosa, 2006). However, coagulationflocculation remains difficult to optimise due to highly variable population densities, morphologies and cell motility as well as interferences by algogenic organic matter (AOM) and, consequently, flotation can be rendered ineffective (Henderson et al. 2010a; Pieterse and Cloot, 1997). This can lead to a number of downstream problems such as turbidity breakthrough, filter clogging (Buisine and Oemcke, 2003), the presence of toxins (AlTebrineh et al. 2010) and the formation of harmful disinfection by-products on disinfection (Chen et al. 2008). Hence, to avoid treatment problems during algal and cyanobacterial blooms, further optimisation of the DAF process is required.

Similar to influent particles and colloids, DAF microbubbles are negatively charged, likely due to asymmetric dipoles of water molecules at bubble gas liquid interfaces (Oliveira and Rubio, 2011). The manipulation of the bubble surface charge, as opposed to that of particles, has received attention as an alternative to coagulation-flocculation (Han et al. 2006; Henderson et al. 2009). Specifically, controlling the bubble surface charge in DAF has been investigated via two methods: 1) Altering the ion content or $\mathrm{pH}$ of water in which bubbles are introduced (Han et al. 2006), or 2) by using a chemical additive dosed into the air saturated water stream (Henderson et al. 2008c, 2009; Karhu et al. 2014; Malley, 1995; Oliveira and Rubio, 2012). For example, Karhu et al. (2014) recently demonstrated the use of modified-bubbles for the treatment of oil-inwater emulsions using cetyl trimethylammonium bromide (CTAB), poly(diallyldimethyl ammonium chloride) (polyDADMAC) and epichlorohydrin-dimethylamine copolymer (Epi-DMA). In this case, CTAB was found to perform poorly in water treatment; however, using polyDADMAC and Epi-DMA as bubble modifiers resulted in $>99 \%$ removal of hydrophobic particles. In the treatment of algae- and cyanobacteria-laden water, Henderson et al. (2008c, 2010b) used a range of surfactants and water treatment polymers to modify bubble surfaces and subsequently float unflocculated cells by dosing chemicals into the recycle stream. When using surfactants (Henderson et al. 2008c), it was found that cell removal for a range of species matched modelled data, reaching a maximum of $64 \%$ removal of Microcystis aeruginosa. Interestingly, when using polyDADMAC (Henderson et al. 2010b), cell removals for the same M. aeruginosa strain reached $98 \%$, indicative of process enhancement via polymer bridging. However, this was not achieved with other species, attributed to competing AOMpolymer and polymer-bubble interactions. Moreover, the positive zeta potential in the treated water was suggestive of high polymer concentrations which are also undesirable.

It has been suggested that more robust flotation of cells may be possible by combining the attributes of both the surfactants and polymers to facilitate greater adherence to bubbles, achieved by incorporating hydrophobic components in a cationic polymer (Henderson et al. 2010b). The development of water treatment polymers has generally targeted higher molecular weight, branched water soluble polymers with no groups that would be conventionally identified as hydrophobically functional (Bolto and Gregory, 2007). Hence, as far as the authors are aware, there have not been any polymers designed to adhere to bubble surfaces in DAF. This research therefore investigates the application of a range of specifically designed hydrophobicallyassociating cationic polymers, in comparison with commercially available polyDADMAC, for the alteration of bubble surface properties in DAF - a process termed 'PosiDAF'.

The aim of this study was to investigate the impact of increasing polymer hydrophobic functionality on the efficacy of bubble coating and link results with the presence of polymer residuals in PosiDAF treated effluent. To achieve this aim, $M$. aeruginosa cells and associated AOM were used as model contaminants. The cell separation obtained using conventional coagulation-flocculation and DAF was also assessed for comparison. The optimal polymer functionalisation for the modification of bubble surfaces was investigated and the mechanisms of interaction between the bubbles, functionalised polymers, cells and AOM discussed.

\section{Materials and methods}

\subsection{Hydrophobically functionalised polymers}

Homopolymers of 2-(N,N-dimethylamino)ethyl methacrylate (DMAEMA) (Aldrich, Australia) were first synthesised as a cationic backbone, controlling the polymer molecular weight by varying the concentration of free radical initiator, azobisisobutyronitrile (AIBN), in a classical free radical polymerisation. DMAEMA was selected as it can be polymerised under 
mild conditions, produces linear polymers and can be easily functionalised. Three base polymers of high, medium and low molecular weights were synthesised and further functionalised by quaternising the tertiary amines with iodomethane, 1bromopentane, 1-bromodecane or 1-bromopentadecane at a range of concentrations. This resulted in increased cationic charge and associated hydrophobic pendant groups. The resulting 36 synthesised polymers were named according to their molecular weight (L, M and $\mathrm{H}$ for low medium and high molecular weight, respectively), the hydrocarbon chain length of the quaternising alkyl halide (C1, C5, C10 and C15, indicating number of carbons) and the concentration of alkylhalide used in the quaternisation reaction $(1, \mathrm{~m}$ and $\mathrm{h}$ for low (10\%), medium (50\%) and high (75\%) conversions, respectively). For example, a low molecular weight polymer with a high concentration of 1-bromopentane was designated LC5-h.

Analysis of the polymers included measuring the charge density using a PCD-04 Travel Charge Demand Analyser (BTG, Switzerland) and the surface tension using a NIMA Surface Tensiometer equipped with a du Noüy ring (Biolin Scientific, Sweden). In this study, nine of the 36 functionalised polymers were selected for investigation to include low, median and high surface tensions in each molecular weight range (Table 1). Pictorial representations can be found in the Table S1.

\subsection{Commercially available chemicals}

Cetyl trimethylammonium bromide (CTAB) (Sigma Aldrich, Australia) and low molecular weight $\left(\mathrm{MW}_{\mathrm{w}} 100-200 \mathrm{kDa}\right)$ poly(diallyldimethylammonium chloride) (polyDADMAC) (Sigma Aldrich, Australia) were used as standard commercially available chemicals to compare the performance of the synthesised polymers. In the conventional coagulationflocculation-DAF experiments, aluminium sulphate (Sigma Aldrich, Australia) was used as a coagulant.

\subsection{Cyanobacteria}

M. aeruginosa (CS-564/01) was obtained from the Commonwealth Scientific and Industrial Research Organisation

Table 1 - Synthesised polymers identified for use in PosiDAF; accompanying data includes polymer stoichiometric quaternisation percentages (as determined by NMR), charge density and surface tension.

\begin{tabular}{lrcc} 
Sample name & $\begin{array}{c}\text { Stoichiometric } \\
\text { quaternisation }\end{array}$ & $\begin{array}{c}\text { Charge } \\
\text { density } \\
\left(\mathrm{meq} \mathrm{g}^{-1}\right)\end{array}$ & $\begin{array}{c}\text { Surface } \\
\text { tension } \\
\left(\mathrm{mN} \mathrm{m}^{-1}\right. \\
\text { at 1 mg L }\end{array}$ \\
\hline LC5-1 & $5 \%$ & 1.20 & 45.6 \\
LC5-m & $21 \%$ & 2.41 & 54.3 \\
LC10-h & $40 \%$ & 2.88 & 69.5 \\
MC1-m & $42 \%$ & 3.44 & 66.2 \\
MC5-1 & $2 \%$ & 1.91 & 44.1 \\
MC10-h & $34 \%$ & 2.45 & 56.8 \\
HC1-m & $35 \%$ & 3.01 & 56.8 \\
HC10-h & $49 \%$ & 2.76 & 69.0 \\
HC15-1 & $8 \%$ & 1.38 & 41.1 \\
polyDMAEMA & $0 \%$ & 1.21 & 44.0 \\
polyDADMAC & $100 \%$ & 6.64 & 71.9 \\
\hline
\end{tabular}

(CSIRO) Australian National Algae Culture Collection, Hobart, Australia, and recultured in MLA media (Bolch and Blackburn, 1996). Cultures were subjected to a $16 / 8 \mathrm{~h}$ light/dark cycle at $21^{\circ} \mathrm{C}$, in a $500 \mathrm{~L}, \mathrm{PG} 50$ incubator with a photosynthetic photon flux output of $600 \pm 60 \mu \mathrm{mol} \mathrm{m}^{-2} \mathrm{~s}^{-1}$ (Labec, Australia). Cultures were grown in $100 \mathrm{~mL}$ batches in $250 \mathrm{~mL}$ conical flasks and agitated frequently to ensure homogeneity of the cultures. Cells were harvested at the end of the exponential growth phase, as determined by cell counting via a Leica DM500 light microscope (Leica Microsystems Ltd, Switzerland) and a haemocytometer. An example of a growth curve can be found in the Supplementary Information (Figure S1). Cell size was measured using a Mastersizer 2000 (Malvern, UK), charge demand with a Mütek PCD-04 particle charge detector (BTG, Switzerland), zeta potential using a Zetasizer Nano ZS (Malvern, UK with a specified zeta potential measurement range of $3.8 \mathrm{~nm}-100 \mu \mathrm{m})$ and AOM concentration using a TOC-VCSH Analyser (Shimadzu, Australia).

\subsection{Conventional flotation jar testing}

A DAF Batch Tester, Model DBT6 (EC Engineering, Canada), was used for conventional flotation experiments incorporating the coagulation-flocculation process. Cultured cells were diluted to $7.5 \times 10^{5}$ cells $\mathrm{mL}^{-1}$ with Milli-Q water buffered with $0.5 \mathrm{mM} \mathrm{NaHCO}_{3}$ and brought to an ionic strength of $1.8 \mathrm{mM}$ using $\mathrm{NaCl}$ to facilitate comparability with previous studies (Henderson et al. 2008c, 2009, 2010b). The saturated water consisted of Milli-Q water also containing $0.5 \mathrm{mM}$ $\mathrm{NaHCO}_{3}$ and made up to an ionic strength of $1.8 \mathrm{mM}$ with $\mathrm{NaCl}$ adjusted to $\mathrm{pH}$ 7. Industrial grade air was used to pressurise the saturator to $450 \mathrm{kPa}$. Coagulation was performed by adding aluminium sulphate to the jar and rapidly mixing for $180 \mathrm{~s}$ at $200 \mathrm{rpm}$. Immediately following the addition of coagulant, the $\mathrm{pH}$ was adjusted to $\mathrm{pH} 7$ using $1 \mathrm{M} \mathrm{HCl}$ and $1 \mathrm{M} \mathrm{NaOH}$ solutions. A pH210 Microprocessor pH Meter (Hanna Instruments, USA) was used to monitor the $\mathrm{pH}$ during rapid mixing. The samples were then flocculated for $10 \mathrm{~min}$ at $30 \mathrm{rpm}$ followed by flotation for $10 \mathrm{~min}$ with an equivalent recycle ratio of $10 \%$, as per typical DAF operation (Edzwald, 2010). Treated water analysis included measuring the cyanobacteria cell concentration, achieved by cell counting and zeta potential analysis (as described in Section 2.3). Each analysis was conducted in triplicate.

\subsection{Bubble charge measurements}

Bubble surface charge was measured to determine whether the polymers altered the surface properties of the bubbles. Measurements were undertaken by the School of Chemical Engineering at the University of Queensland. A Microelectrophoresis Apparatus Mk II (Rank Brothers Ltd., UK), consisting of a rectangular cell $(10 \mathrm{~mm} \times 1 \mathrm{~mm})$ and platinum electrodes, was used in the measurement of microbubbles. The generation of microbubbles and measurement was adapted from that described by Qu et al. (2009). Specifically, nitrogen was dissolved into a Milli-Q solution comprising $0.5 \mathrm{mM} \mathrm{NaHCO}_{3}$ and made up to an ionic strength of $1.8 \mathrm{mM}$ with $\mathrm{NaCl}$, corrected to $\mathrm{pH} 7$ with $1.6 \mathrm{mg} \mathrm{L}^{-1}$ of polymer, at $450 \mathrm{kPa}$ by leaving overnight. The polymer concentration was based on saturator 
concentrations applied in PosiDAF jar testing. About $100 \mathrm{~mL}$ of the oversaturated solution was introduced to the glass cell of the microelectrophoretic unit. At room pressure, microbubbles that formed were subject to an electrical field of $40 \mathrm{~V} / \mathrm{m}$. The motion of the bubbles was then recorded with a CCD camera and their electrophoretic mobilities and zeta potentials were calculated using the von Smoluchowski equation (Hunter, 1981), for which 60 to 100 bubble measurements were obtained per polymer tested.

\subsection{PosiDAF jar testing}

The same DAF Batch Tester, Model DBT6 (EC Engineering, Canada), and cell suspensions were used for PosiDAF jar testing as in Section 2.4. The recycle water composition identical to that described in Section 2.4 except that the various polymers at a range of concentrations up to $3 \mathrm{mg} \mathrm{L}^{-1}$ were now added to the buffered solution. Industrial grade air was again used to pressurise the saturator to $450 \mathrm{kPa}$ but, in these experiments, an equivalent recycle ratio of $20 \%$ was applied to ensure a high bubble to particle ratio was maintained given that coagulation-flocculation would not be applied to lower particle numbers. Flotation was conducted for 10 min prior to sampling of the treated effluent without any coagulation-flocculation. Treated water analysis was undertaken as previously described for conventional flotation experiments. Zeta potential measurements were anticipated to give an indication of the presence of polymer in the treated water, as any residual cationic polymer will either complex with or adsorb onto oppositely charged colloids/particles or remain free in solution. Note that the hydrodynamic diameters of the polymers in solution were found to be 12.2-1130 nm (Table S2), greater than the $3.8 \mathrm{~nm}$ zeta potential measurement limit of the instrument. Overall, the presence of cations is expected to reduce the magnitude of the measured negative charge in the treated water.

\section{Results}

\subsection{Microcystis aeruginosa Characterisation}

On microscopic evaluation of M. aeruginosa cultures harvested at the end of the exponential growth phase, the cells were found to be spherical and unicellular with an average

Table 2 - Microcystis aeruginosa cell properties.

\begin{tabular}{|c|c|c|}
\hline Attribute & CS-564/01 & CCAP $1450 / 3$ \\
\hline Morphology & Spherical & Spherical \\
\hline Diameter $(\mu \mathrm{m})$ & $3.0 \pm 0.7$ & $5.4^{a}$ \\
\hline $\begin{array}{l}\text { Cell concentration } \\
\text { (cells } \mathrm{mL}^{-1} \text { ) }\end{array}$ & $2.1 \times 10^{7} \pm 2 \times 10^{6}$ & - \\
\hline $\mathrm{AOM}\left(\mathrm{mg} \mathrm{C}\right.$ cell $\left.^{-1}\right)$ & $8.04 \times 10^{-10} \pm 4.4 \times 10^{-11}$ & $10 \times 10^{-10 a}$ \\
\hline Zeta potential (mV) & $-31.6 \pm 1.6$ & $-19.8^{b}$ \\
\hline $\begin{array}{l}\text { Charge density } \\
\left.\text { (meq cell }{ }^{-1}\right)\end{array}$ & $-1.51 \times 10^{-9} \pm 7 \times 10^{-11}$ & $-2.0 \times 10^{-12 a}$ \\
\hline
\end{tabular}

diameter of $3.0 \pm 0.7 \mu \mathrm{m}$ (Table 2). The charge density and zeta potential of the cell culture were determined to be $-1.51 \times 10^{-9} \pm 7 \times 10^{-11} \mathrm{meq}$ cell $^{-1}$ and $-31.6 \pm 1.6 \mathrm{mV}$, respectively. Average cell concentration and concentration of AOM at this phase of growth was $2.1 \times 10^{7} \pm 2 \times 10^{6}{\text { cells } \mathrm{mL}^{-1}}^{-1}$ and $8.04 \times 10^{-10} \pm 4.4 \times 10^{-11} \mathrm{mg} \mathrm{C}$ cell $^{-1}$, respectively (Table 2). Notably, Henderson et al. (2008b) found that cell sizes obtained for the UK strain of M. aeruginosa (CCAP 1450/3) used in PosiDAF research were larger $(5.4 \mu \mathrm{m})$ and had a much lower charge density per cell of $2.0 \times 10^{-12}$ meq cell ${ }^{-1}$. If it is assumed that all AOM was associated at the cell surfaces, this indicates that the Australian strain (CS-564/01) had a much more negative cell surface charge density at -57 meq $\mathrm{m}^{-2}$ compared to $-0.04 \mathrm{meq} \mathrm{m}^{-2}$ for CCAP 1450/3 (Henderson et al. 2008b). Similar to the surface charge density, the zeta potential of the Australian strain was also more negative.

\subsection{Cell removal with conventional DAF}

Conventional DAF with coagulation-flocculation pre-treatment upstream of flotation resulted in high cell removal efficiencies that were dependent on effective coagulation (Fig. 1). For example, it was observed that a dose of $1 \mathrm{mg} \mathrm{L}^{-1}$ as $\mathrm{Al}$ (or $\left.11.1 \mathrm{mg} \mathrm{L}^{-1} \mathrm{Al}_{2}\left(\mathrm{SO}_{4}\right)_{3} \bullet 14 \mathrm{H}_{2} \mathrm{O}\right)$ was required to achieve cell removals greater than $95 \%$, coinciding with a lowering of the magnitude of the zeta potential. At a dose of $5 \mathrm{mg} \mathrm{L}^{-1}, \mathrm{a}$ maximum cell removal of $99 \%$ was obtained and the formation of large flocs observed (Fig. 2).

\subsection{Modified bubble properties}

Bubbles coated with hydrophobically functionalised polyDMAEMA were confirmed to be cationic at $\mathrm{pH} 7$, with zeta potentials ranging from between $+38.6 \mathrm{mV}$ and $+63.8 \mathrm{mV}$. These values were comparable with the charge of bubbles modified with polyDMAEMA homopolymer, polyDADMAC and $\mathrm{CTAB}$ of $+39 \pm 10 \mathrm{mV},+44 \pm 9 \mathrm{mV}$ and $+44 \pm 7 \mathrm{mV}$, respectively (Fig. 3). It was found that zeta potentials observed in the current study were consistently more positive than those observed in prior work. For example, Cho et al. (2005) used a range of cationic surfactants to modify nanobubbles,

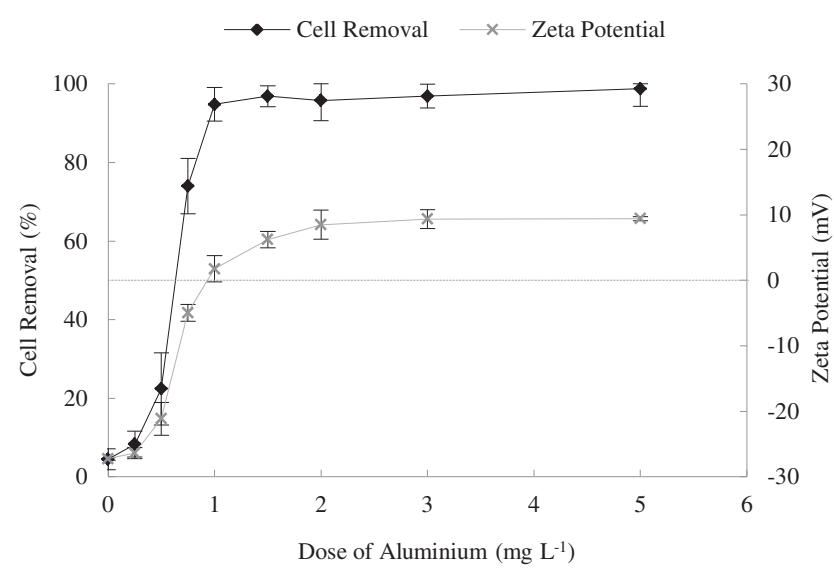

Fig. 1 - Conventional DAF with coagulation-flocculation pretreatment; dose is reported as concentration as $\mathrm{Al}$. 


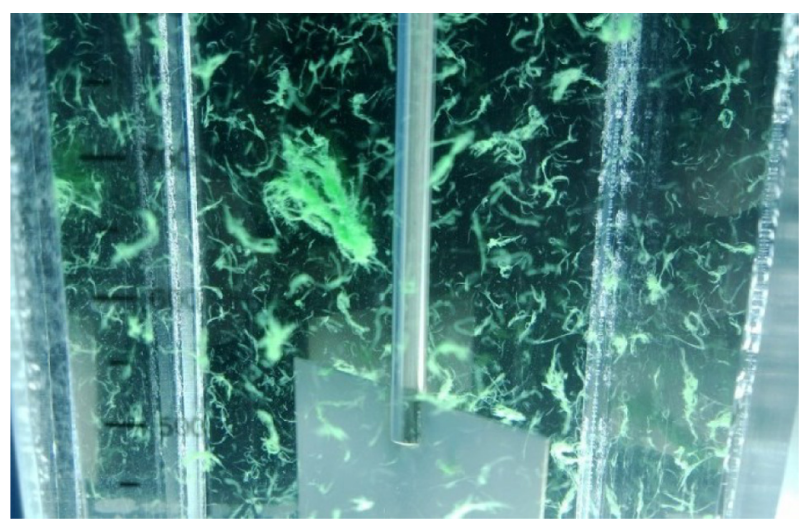

Fig. 2 - Observation of flocs formed after coagulation of $\mathbf{M}$. aeruginosa with alum at a dose of $1 \mathrm{mg} \mathrm{L}^{-1}$ as Al.

resulting in bubbles with a maximum zeta potential of $+30 \mathrm{mV}$ at pH 7. Similarly, Han et al. (2006) generated bubbles with a zeta potential of $+30 \mathrm{mV}$ using aluminium hydroxide, although high standard deviations were observed.

Overall, bubble zeta potential did not vary to the same extent as polymer charge density. It was observed that the modification of bubbles with polymers quaternised with a high concentration of $\mathrm{C} 10$ groups resulted in bubbles with less positive zeta potentials for each of the molecular weight ranges (specifically, polymers LC10-h, MC10-h and HC10-h). It was found that LC10-h had a statistically different zeta potential compared to both LC5-1 and LC5-m (P-value <0.05), whereas the zeta potential values of LC5-m and LC5-m were found to be statistically the same ( $P$-value 0.16$)$. Corresponding observations were made for MC10-h and HC10-h in the medium and high molecular weight polymer groups, respectively. Bubbles modified with LC10-h, MC10-h and HC10-h had more negative surface zeta potentials, suggesting that less charge and thus polymer was adsorbed per bubble. Though polymers with larger hydrophobic groups were synthesised

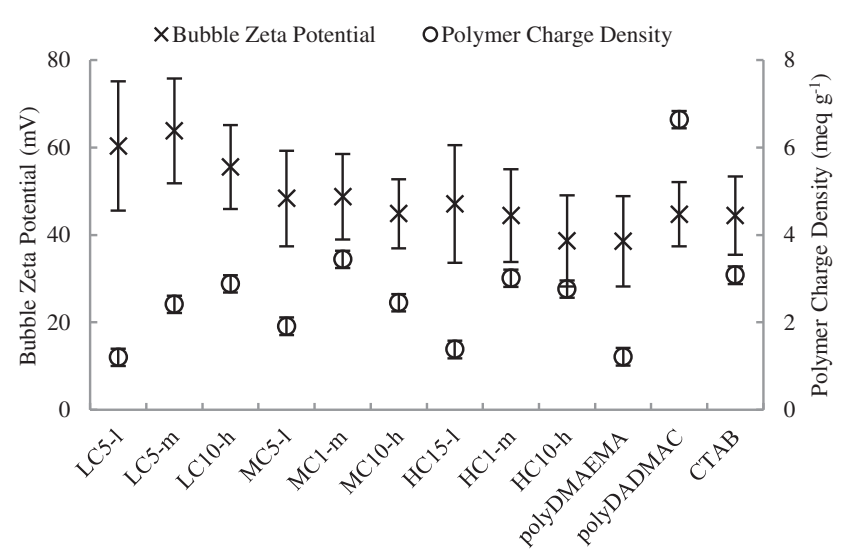

Fig. 3 - Average bubble zeta potentials for the selection of polymers, polyDADMAC and CTAB $(\times)$ and respective charge density of the polymer used in the test $(O)$; zeta potential measurements were conducted on solutions made up to $1.8 \mathrm{mM}$ of $\mathrm{NaCl}$ and $0.5 \mathrm{mM}$ of $\mathrm{NaHCO}$, corrected to $\mathrm{pH} 7$ with $1.6 \mathrm{mg} \mathrm{L}^{-1}$ of polymer.
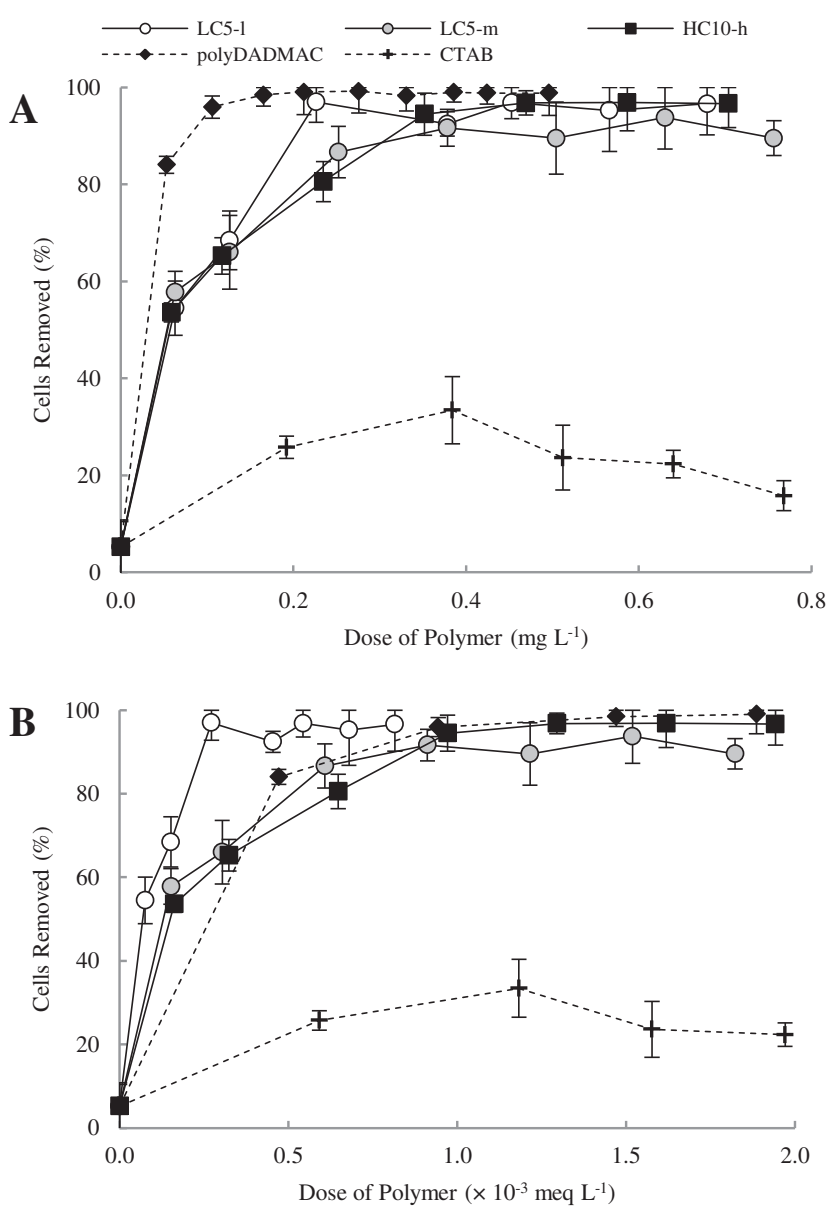

Fig. 4 - Dose response curves for three of the nine polymers and CTAB in comparison to polyDADMAC and CTAB - graphs show cell removal versus dose as (A) polymer mass and (B) dose as charge - the results for all polymers are presented in Table S3.

(C15), samples with higher stoichiometric quaternisations $(>10 \%)$ formed gels and thus could not be analysed in solution.

On examining the average zeta potential for each of the molecular weight groups, it was revealed that lower molecular weight polymers resulted in more positive bubble zeta potentials compared to the medium and high molecular weight polymers ( $P$-values 0.016 and 0.025 , respectively). For example, the average bubble zeta potentials for each of the polymer molecular weight groups (low, medium and high) were $+60 \pm 13 \mathrm{mV},+47 \pm 10 \mathrm{mV}$ and $+42 \pm 12 \mathrm{mV}$, respectively.

\subsection{Cell removal using PosiDAF}

Results from jar tests that were conducted using the nine synthesised polymers to modify bubble surfaces demonstrated that cell removals in excess of $93 \%$ were achievable for each polymer tested when applying doses of $0.3 \mathrm{mg} \mathrm{L}^{-1}$, without coagulation (Fig. 4A). With a maximum cell removal of $99 \%$, using $0.3 \mathrm{mg} \mathrm{L}^{-1}$ of polyDADMAC, PosidAF has the same cell removal efficiency as conventional DAF (Fig. 1). The 
resultant dose response curves for all polymers are presented in the Supplementary Information (Table S3). Overall, the dose response curves increased to greater than $90 \%$ cell removal, with no decrease of cell removal observed even at the highest doses of polymer used in this test. All dose response curves were similar despite variations of polymer charge densities and associated hydrophobic groups as indicated in Table 1. Comparing the polymers in terms of their charge dose demonstrated similarity in the removal efficiencies for polyDADMAC and synthesised polyDMAEMA samples with medium and high concentrations of quaternised residuals, specifically LC5-m, LC10-h, MC1-m, MC10-h, HC1-m and HC10-h (select examples in Fig. 4B and full dataset in Table S3). However, polymers with low concentrations of quaternised residuals (LC5-1, MC5-l and HC15-1) resulted in greater removal efficiencies at low charge concentrations. As an example, the charge dose response curve for LC5-l is compared to the curves for LC5-m, HC10-h, polyDADMAC and CTAB in Fig. 4B. It can be seen that for a polymer dose of approximately $0.3 \times 10^{-3}$ meq $\mathrm{L}^{-1}$, cell removal achieved by PosiDAF with LC5-m and HC10-h was $66 \pm 6 \%$, whereas with LC5-1, $97 \pm 4 \%$ was achieved. This indicates that polymer bridging is a dominant mechanism and elevated charge may not be necessary in establishing polymer-particle attachments. However, it is known that low charge density polymers have different neutralisation effects in water: Kam and Gregory (2001) showed that polymers with a charge density of

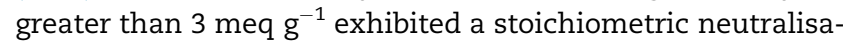
tion of anionic humic substances, where 1 meq of polymer could neutralise 1 meq of humic substances. This was not true for polymers with charge densities less than 3 meq $^{-1}$, where less than 1 meq of polymer could neutralise 1 meq of humic substances.

Similar to the PosiDAF jar tests undertaken with the hydrophobically functionalised polymer, jar tests conducted with polyDADMAC revealed that cell removal was again high, with up to $99 \%$ removal achieved at doses above $1.0 \times 10^{-3} \mathrm{meq} \mathrm{L}^{-1}$. Using a UK strain of M. aeruginosa (CCAP 1450/3), Henderson et al. (2009) found that $95 \%$ removal could be achieved at a dose of $2.4 \times 10^{-3} \mathrm{meq} \mathrm{L}^{-1}$. This study therefore indicates that the required polymer dose was strain dependent. To demonstrate this, the dose was normalised to charge dose per cell charge. In this work, the optimal dose was found to be 0.9 meq polyDADMAC per meq M. aeruginosa whereas Henderson et al. (2009) reported the optimal dose to be 1.7 meq polyDADMAC per meq M. aeruginosa for CCAP 1450/ 3. A major difference between these strains was the cell size, where CS-564/01 cells were nearly half the diameter of CCAP 1450/3 cells ( $3.0 \mu \mathrm{m}$ versus $5.4 \mu \mathrm{m}$, respectively). Considering this, the relative dose per cell surface area is $67 \%$ greater for CS-564/01 than for CCAP 1450/3 at the same cell concentration. In addition, for the same comparison, the charge density was also found to be significantly greater for CS-564/01 (Henderson et al., 2010a). Differences of this scale between species have previously been reported (Henderson et al. 2008a, 2010a); the observation that such differences in charge density can also occur between different strains of the same species is an important consideration.

The highest cell removal obtained using CTAB as the bubble modifier ( $33 \pm 7 \%$ with a dose of $1.18 \times 10^{-3} \mathrm{meq} \mathrm{L}^{-1}$, Fig. 4 ) was found to be much lower than that obtained for polymers and obtained by Henderson et al. (2008c) (64\% with a dose of $2.2 \times 10^{-3}$ meq L $^{-1}$ ). However, both the results obtained in this study and by Henderson et al. (2008c) are comparable with modeled results obtained using the white water model performance equation (Haarhoff and Edzwald, 2004) (Equation S1). For example, assuming $100 \%$ attachment efficiency, it was determined that the modeled cell removal was $30 \%$ for a particle size of $3.0 \mu \mathrm{m}$, and $64 \%$ for a particle size of $5.4 \mu \mathrm{m}$.

Interestingly, unlike observations made by Henderson et al. (2009), large bubble-cell networks were observed to develop during the $10 \mathrm{~min}$ flotation period of CS-564 (Fig. 5A), creating a stable, cell-rich float (Fig. 5B). These networks formed rapidly after the introduction of the recycle stream to become clearly visible to the naked eye and may be the result of extended bridging which has in turn aided cell removal. The formation of such structures may be attributed to large biopolymers in AOM present in algae and cyanobacteria systems (Henderson et al. 2010a) which can influence the action of the polymers PosiDAF. For example, it has been demonstrated that polymers preferentially interact with AOM over cells (Haarhoff and Cleasby, 1989) and dissolved matter over other particles (Lurie and Rebhun, 1997). Furthermore, in the case of CCAP1450/3 (Henderson et al. 2010b), polymer-AOM interactions were suggested as a mechanism to create favourable flotation conditions for cells.

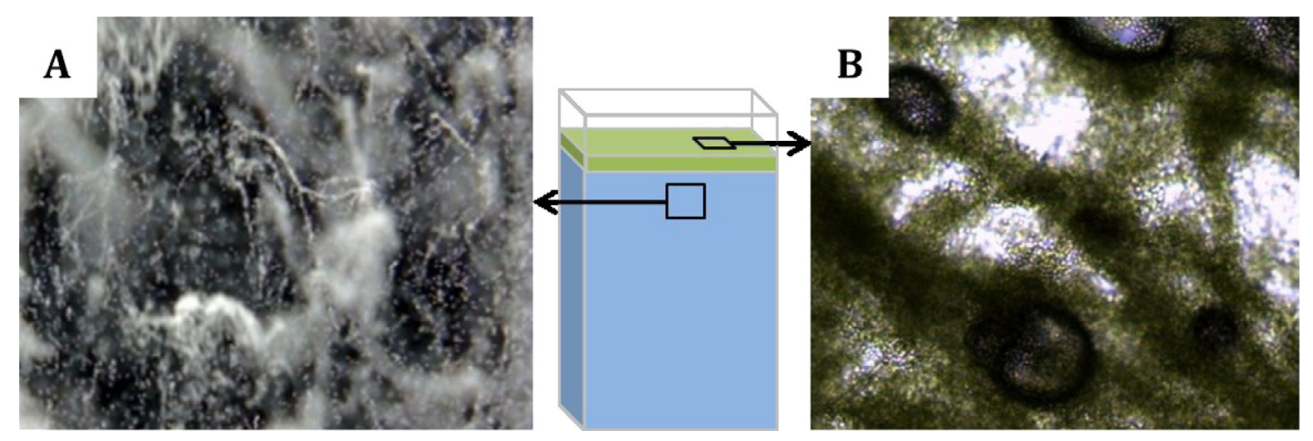

Fig. 5 - Visual observations from PosidAF using $2 \times 10^{-3} \mathrm{meq} \mathrm{L}^{-1}$ of polyDADMAC - (A) a photograph of rising bubble networks beneath the float layer, observed from the side of the jar after the introduction of saturated water ( $26 \mathrm{~mm}$ lens) and (B) A microscope image of the float after a jar test $(10 \times$ magnification). 


\subsection{Charge measured in the PosiDAF treated water}

The charge of particles or dispersed/dissolved macromolecules measured in the treated water after jar tests using the nine synthesised polymers was highly variable, ranging from $-44.6 \mathrm{mV}$ to $+12.7 \mathrm{mV}$. A full set of these results can be found in the Supplementary Information (Table S3). Zeta potential dose response curves for LC5-1, LC5-m and HC10-h are displayed as an example of this and compared to CTAB and polyDADMAC in Fig. 6. For all polymers, increasing the polymer dose resulted in a decrease in the magnitude of the negative zeta potentials in the treated water to some degree (Table S3). Of the polymers tested, those modified with high concentrations of highly hydrophobic groups, specifically, LC10-h, MC10-h and HC10-h, resulted in the most negative resultant zeta potentials upon jar testing and in fact retained negative zeta potentials over the range of doses applied. Conversely, polymers with the fewest quaternised groups resulted in positive resultant zeta potentials at lower charge doses than the other polymers (e.g. LC5-l in Fig. 6). With highly cationic polymers, such as polyDADMAC and LC5-m, zeta potentials became positive with increasing polymer dose. After a jar test, the remaining cells, AOM and polymer in the treated water contribute to the zeta potential. As charge measurements can be used to determine polymer or colloid concentrations (Kam and Gregory, 1999), a positive zeta potential is indicative of relative residual polymer concentration when contrasted with results of other polymers with similar cell removals. It was found that higher molecular weight polymers resulted in negative zeta potentials at much greater doses than that needed for optimal cell removal (Table S3). Similar observations have been made before when using PosidAF (Henderson et al. 2010b) and for conventional DAF (Gehr and Henry, 1982). CTAB demonstrated highly anionic zeta potentials at all doses in this study, which can be attributed to its ability to congregate at air-water interfaces. Synthesised polymers with the highest degree of functionalization, LC10-h, MC10-h and HC10-h, displayed the most negative zeta potentials in PosiDAF treated effluent (Table S3). In particular, the zeta potentials obtained from

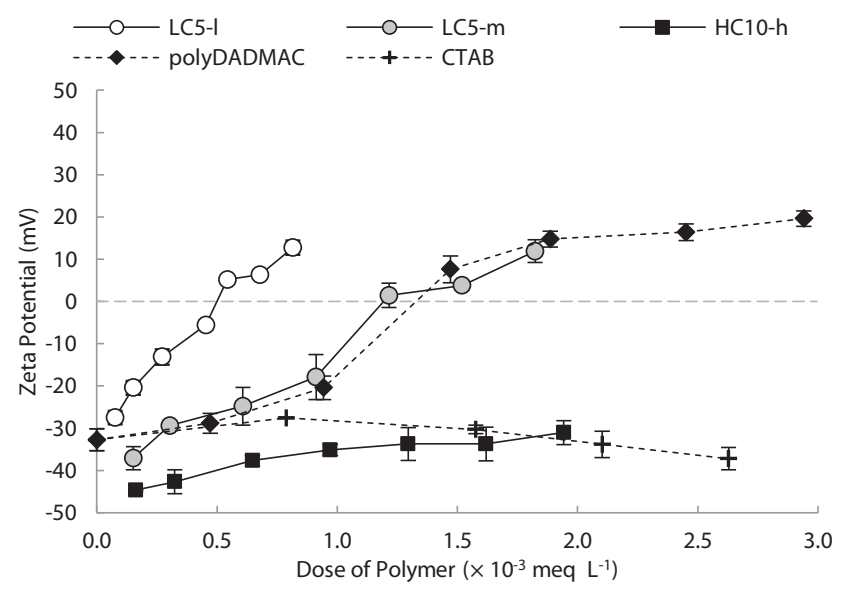

Fig. 6 - Dose response of HC10-3, LC5-1, LC5-2, polyDADMAC and CTAB in terms of zeta potential - the results for all polymers are presented in Table $\mathrm{S} 3$. tests using HC10-h were most negative and very similar to that of CTAB, suggesting the strongest adhesion to the bubble surface was achieved when using this polymer.

\section{Discussion \\ 4.1. Bubble coating with hydrophobically functionalised polymers}

The surfaces of microbubbles in water have been found to be negatively charged under a range of $\mathrm{pH}$ conditions (Elmallidy et al. 2008; Han et al. 2006). At pH 7, the zeta potential of a micro-bubble in water is approximately -25 to $-60 \mathrm{mV}$ (Oliveira and Rubio, 2011; Yang et al. 2001), though the value can be altered depending on background ionic conditions (Oliveira and Rubio, 2011; Yang et al. 2001). Bubbles modified with CTAB and polyDADMAC resulted in positive bubbles with little variation in the magnitude of their zeta potentials at $\mathrm{pH}$ 7. This is in agreement with literature for CTAB (Yoon and Yordan, 1986); however, the study of the effect of various cationic polymers is limited to commercially available polymers (Oliveira and Rubio, 2011). With all synthesised polymers used in this work, positively charged bubbles were generated; however, the bubble zeta potential was observed to shift to less positive values with increases in a) polymer hydrophobic functionality and b) polymer molecular weight (Fig. 3).

With respect to hydrophobic functionality in synthesised polymers, the association of hydrophobic pendant groups to cationic polyDMAEMA was expected to enhance electrostatic polymer interaction at the bubble surfaces by hydrophobic association (Bütün et al. 2001). Counterintuitively, the polymers with high concentrations of large hydrophobic groups (LC10-h, MC10-h and HC10-h) were observed to have a less positive bubble zeta potential in comparison to polymers of similar molecular weight, despite these polymers having greater charge densities than the other DMAEMA-based polymers (Table 1). Assuming that this was the result of lower charge concentrations at the bubble surface, it is suggested that the polymer attached to the bubble surface in a flatter conformation than other polymers due to increased hydrophobicity, similar to the adsorption of hydrophobic polymers onto hydrophobic surfaces (Jamadagni et al. 2009). This would result in any given unimer occupying a larger surface area therefore limiting the attachment of other unimers via steric interactions.

With respect to polymer molecular weight, the shift in bubble zeta potential to less positive values was surprising as Aoki and Adachi (2006) had observed that the electrophoretic mobility of polystyrene latex particles with adsorbed fully quaternised polyDMAEMA was constant, regardless of the polymer molecular weight. However, the adsorption of polymers onto bubbles may be inhibited not only by steric interferences in the case of high molecular weight polymers, but also by electrostatic repulsion in areas where cationic unimers have adsorbed to form areas of high charge concentration. It is anticipated that the extension of a low molecular weight polymer from the surface of a bubble will be less than that of high molecular weight polymers (Henderson et al. 2010b; Napper, 1983), therefore it may be that less steric interaction 
would be encountered and more polymers (and charge) could occupy the same area on a microbubble surface. It is acknowledged, however, that further complications can arise in modified-bubble measurements due to differing polymer concentrations on bubble surfaces in a single system; for example, Oliveira and Rubio (2011) observed that randomly measured bubbles do not carry the same charge in a given system.

With respect to the toxicity of polymers in water treatment, it is generally accepted that the polymers with greater cationicity are more toxic (Bolto and Gregory, 2007). The inclusion of hydrophobic groups to a cationic polymer can increase antibacterial activity at high concentrations (van de Wetering et al. 2000); however, it has been long recognised that polymers with surfactant-like residuals are much less toxic than surfactants (Schmolka, 1977).

For surfactants, surface activity measurements can be related to packing density of the molecule at the air-water interface (Henderson et al. 2008c; Rosen and Milton, 1978). The positive bubble zeta potential measurements observed in this study (Fig. 3) and in other studies (Malley, 1995; Oliveira and Rubio, 2011) indicate polymer adsorption at the bubble surface. However, highly cationic polymers (specifically LC10-h and HC10-h) had surface tensions similar to that of pure water at $72 \mathrm{mN} \mathrm{m}^{-1}$ (Table 1) despite the hydrophobic functionality. Similarly, in an investigation of polymers with low polydispersity at an air-water interface, Matsuoka et al. (2004) also found that charged polymers exhibited 'non-surface activity' despite the presence of hydrophobic groups. Polymer accumulation at the air-liquid interface is theorised to occur as hydrogen bonding with $\mathrm{OH}^{-}$or $\mathrm{H}^{+}$groups (Yang et al. 2001), which translates to air-liquid surface adsorption without surface penetration. This has been confirmed with X-ray reflectance, demonstrating that both hydrophilichydrophobic random copolymers and hydrophilic homopolymers did adsorb at the air-liquid interface in solution, despite demonstrating no change in surface tension over a large concentration range (Matsuoka et al. 2012). This means that packing density cannot be estimated using polymer surface activity and therefore further research is required to gain a fuller understanding of the mechanism for polymer adsorption and packing at a bubble surface.

\subsection{Mechanisms of cell removal in PosiDAF}

When chemicals are applied to bubble surfaces as opposed to particles and colloids, the flotation of particles from water is dependent on their effective interaction with bubbles. In this work and previous research (Henderson et al. 2008c), the use of CTAB resulted in cell removals comparable to that predicted by theory and were thus dependent on cell size (Haarhoff and Edzwald, 2004). The use of polymers for this cyanobacteria system was able to exceed the theoretical particle removal efficiency, demonstrated not only in this research, but on other algae and cyanobacteria (Henderson et al. 2010b) and other synthetic waters (Malley, 1995). A potential mechanism for this enhanced separation, as discussed by Henderson et al. (2010b), is the increase in 'swept volume', whereby the effective surface area of a polymer modified bubble is greater than an unmodified bubble, facilitating further cell attachments. However, as the bridging distances of the polymers used are insignificant compared to cell sizes (200 nm (Henderson et al. 2010b) versus $3.0 \mu \mathrm{m}$, respectively), additional interactions must also occur. During PosiDAF jar tests, the large bubble-cell networks that were observed may be the result of AOM and polymer complexation, resulting in extended bridging lengths that enhanced the capture of cells. This is plausible as it has been shown that polymer preferentially interacts with AOM over cells (Haarhoff and Cleasby, 1989). When considering applications of the process in a water treatment context, the presence of additional organic matter from sources other than algae/ cyanobacteria could alter bubble-polymer-cell interactions as observed in this work. This would require investigation in the appropriate context.

With high cell removals in all polymer samples tested, the PosiDAF polymer performance was indicated by the zeta potential in the treated water. The association of polymers containing a large number of hydrophobic pendant groups, for example polymer HC10-h, may further strengthen polymer adhesion to bubbles. The grouping of the hydrophobic regions can also facilitate bubble nucleation via catalytic effects offered by the hydrophobic zones (Lubetkin, 2003), leading to bubbles formed with polymers in situ. When bubbles are formed in the presence of the polymer, little to no diffusion is required to associate a polymer to its surface. Though the hydrophobically functionalised polymer may not project into solution as readily as a cationic polymer without hydrophobic pendant groups, it is evident that bubble-polymer-AOM-cell suprastructures still form. It was therefore found that the ideal polymer for PosiDAF were those comprising increased proportions of hydrophobic functionalisation of long hydrophobic pendant groups, specifically a carbon chain length of approximately C10.

\section{Conclusions}

The following conclusions have been drawn from this study:

- Use of CTAB, polyDADMAC and all synthesised polymers as bubble modifiers resulted in positively charged bubbles with statistically different zeta potentials and charge characteristics.

- The use of all synthesised polymers resulted in cell removals in excess of $90 \%$ with a maximum of $99 \%$. These removals were comparable to that obtained when using commercial polyDADMAC as a bubble modifier and conventional coagulation-DAF.

- The most negative zeta potentials in PosidAF treated effluent were achieved using synthesised polymer HC10-h, indicating a relatively low polymer concentration in the treated water and therefore stronger bubble attachment. This suggests that higher polyDMAEMA quaternisation/ hydrophobic functionality leads to enhanced bubble attachment.

- Large suprastructures were observed to form, suggesting that polymers and AOM complex, leading to large networks that link bubbles, polymer, AOM and cells, enhancing overall cell capture and thus exceeding removal efficiencies that are predicted by a flotation model. 


\section{Acknowledgements}

This research was supported under Australian Research Council's Linkage Projects funding scheme (project number LP0990189) which included support from SA Water, Veolia Water, United Water, Melbourne Water and Seqwater. For the same project (LP0990189), Dr Rita Henderson was a recipient of an Australian Research Council Post Doctoral Industry Fellowship and Russell Yap was a recipient of an Australian Research Council Postgraduate Award Industry scholarship. In addition, the authors would like to thank Water Research Australia for the PhD top up scholarship (project number 4025-10) provided over the duration of this work.

\section{Appendix A. Supplementary data}

Supplementary data related to this article can be found at http://dx.doi.org/10.1016/j.watres.2014.05.032.

\section{R E F E R E N C E S}

Al-Tebrineh, J., Mihali, T.K., Pomati, F., Neilan, B.A., 2010. Detection of saxitoxin-producing cyanobacteria and anabaena circinalis in environmental water blooms by quantitative PCR. Appl. Environ. Microbiol. 76 (23), 7836-7842.

Aoki, K., Adachi, Y., 2006. Kinetics of polyelectrolyte adsorption onto polystyrene latex particle studied using electrophoresis: effects of molecular weight and ionic strength. J. Colloid Interface Sci. 300 (1), 69-77.

Bolch, C.J.S., Blackburn, S.I., 1996. Isolation and purification of Australian isolates of the toxic cyanobacterium Microcystis aeruginosa Kütz. J. Appl. Phycol. 8 (1), 5-13.

Bolto, B., Gregory, J., 2007. Organic polyelectrolytes in water treatment. Water Res. 41 (11), 2301-2324.

Buisine, F., Oemcke, D., 2003. Seasonal influence of waste stabilisation pond effluent on DAF/F (dissolved air flotation/ filtration) process operation. Water Sci. Technol. 48 (2), 357-364.

Bütün, V., Armes, S.P., Billingham, N.C., 2001. Selective quaternization of 2-(dimethylamino)ethyl methacrylate residues in tertiary amine methacrylate diblock copolymers. Macromol. 34 (5), 1148-1159.

Chen, C., Zhang, X.j., Zhu, L.x., Liu, J., He, W.j., Han, H.d, 2008. Disinfection by-products and their precursors in a water treatment plant in North China: seasonal changes and fraction analysis. Sci. Total Environ. 397 (1-3), 140-147.

Cho, S.-H., Kim, J.-Y., Chun, J.-H., Kim, J.-D., 2005. Ultrasonic formation of nanobubbles and their zeta-potentials in aqueous electrolyte and surfactant solutions. Colloids Surf. Physicochem. Eng. Asp. 269 (1-3), 28-34.

Edzwald, J.K., 2010. Dissolved air flotation and me. Water Res. 44 (7), 2077-2106.

Elmallidy, A.M., Mirnezami, M., Finch, J.A., 2008. Zeta potential of air bubbles in presence of frothers. Int. J. Miner. Process. 89 (1-4), 40-43.

Gehr, R., Henry, J.G., 1982. The adsorption behaviour of cationic polyelectrolytes in dissolved air flotation. Water Sci. Technol. $14(6-7 / 7), 689-704$.

Haarhoff, J., Cleasby, J.L., 1989. Direct filtration of Chlorella with cationic polymer. J. Environ. Eng. 115 (2), 348-366.
Haarhoff, J., Edzwald, J.K., 2004. Dissolved air flotation modelling: insights and shortcomings. J. Water Supply Res. Technol.Aqua 53 (3), 127-150.

Han, M.Y., Kim, M.K., Shin, M.S., 2006. Generation of a positively charged bubble and its possible mechanism of formation. J. Water Supply Res. Technol.Aqua 55 (7-8), 471-478.

Henderson, R.K., Baker, A., Parsons, S.A., Jefferson, B., 2008 a. Characterisation of algogenic organic matter extracted from cyanobacteria, green algae and diatoms. Water Res. 42 (13), 3435-3445.

Henderson, R.K., Parsons, S.A., Jefferson, B., 2008b. Successful removal of algae through the control of zeta potential. Sep. Sci. Technol. 43 (7), 1653-1666.

Henderson, R.K., Parsons, S.A., Jefferson, B., 2008c. Surfactants as bubble surface modifiers in the flotation of algae: dissolved air flotation that utilizes a chemically modified bubble surface. Environ. Sci. Technol. 42 (13), 4883-4888.

Henderson, R.K., Parsons, S.A., Jefferson, B., 2009. The potential for using bubble modification chemicals in dissolved air flotation for algae removal. Sep. Sci. Technol. 44 (9), 1923-1940.

Henderson, R.K., Parsons, S.A., Jefferson, B., 2010a. The impact of differing cell and algogenic organic matter (AOM) characteristics on the coagulation and flotation of algae. Water Res. 44 (12), 3617-3624.

Henderson, R.K., Parsons, S.A., Jefferson, B., 2010b. Polymers as bubble surface modifiers in the flotation of algae. Environ. Technol. 31 (7), 781-790.

Hunter, R.J., 1981. Zeta Potential in Colloid Science : Principles and Applications. Academic Press, London, London.

Jamadagni, S.N., Godawat, R., Garde, S., 2009. How surface wettability affects the binding, folding, and dynamics of hydrophobic polymers at interfaces. Langmuir 25 (22), 13092-13099.

Kam, S.K., Gregory, J., 1999. Charge determination of synthetic cationic polyelectrolytes by colloid titration. Colloids Surf. Physicochem. Eng. Asp. 159 (1), 165-179.

Kam, S.K., Gregory, J., 2001. The interaction of humic substances with cationic polyelectrolytes. Water Res. 35 (15), 3557-3566.

Karhu, M., Leiviskä, T., Tanskanen, J., 2014. Enhanced DAF in breaking up oil-in-water emulsions. Sep. Purif. Technol. 122 (0), 231-241.

Lubetkin, S.D., 2003. Why is it much easier to nucleate gas bubbles than theory predicts? Langmuir 19 (7), 2575-2587.

Lurie, M., Rebhun, M., 1997. In: Ives, K.J., Adin, A. (Eds.), Effect of Properties of Polyelectrolytes on Their Interaction with Particulates and Soluble Organics. Isr, Jerusalem, pp. 93-101.

Malley, J.P., 1995. The use of selective and direct DAF for removal of particulate contaminants in drinking water treatment. Water Sci. Technol. 31 (3-4), 49-57.

Matsuoka, H., Hachisuka, M., Uda, K., Onishi, T., Ozoe, S., 2012. Why ionic amphiphilic block copolymer can be non-surface active comparison of homopolymer, block and random copolymers of poly(styrenesulfonate). Chem. Lett. 41 (10), 1063-1065.

Matsuoka, H., Maeda, S., Kaewsaiha, P., Matsumoto, K., 2004. Micellization of non-surface-active diblock copolymers in water. Special characteristics of poly(styrene)-blockpoly(styrenesulfonate). Langmuir 20 (18), 7412-7421.

Napper, D.H., 1983. Polymeric Stabilization of Colloidal Dispersions. Academic Press, London, London.

Oliveira, C., Rubio, J., 2011. Zeta potential of single and polymercoated microbubbles using an adapted microelectrophoresis technique. Int. J. Miner. Process. 98 (1-2), 118-123.

Oliveira, C., Rubio, J., 2012. Kaolin aerated flocs formation assisted by polymer-coated microbubbles. Int. J. Miner. Process. 106-109 (0), 31-36. 
Pieterse, A.J.H., Cloot, A., 1997. Algal cells and coagulation, flocculation and sedimentation processes. Water Sci. Technol. 36 (4), 111-118.

Qu, X., Wang, L., Karakashev, S.I., Nguyen, A.V., 2009.

Anomalous thickness variation of the foam films stabilized by weak non-ionic surfactants. J. Colloid Interface Sci. 337 (2), 538-547.

Rosen, M.J., Milton, J.R., 1978. Surfactants and Interfacial Phenomena. Wiley, New York, New York.

Schmolka, I., 1977. A review of block polymer surfactants. J. Am. Oil Chem.' Soc. 54 (3), 110-116.

Teixeira, M.R., Rosa, M.J., 2006. Comparing dissolved air flotation and conventional sedimentation to remove cyanobacterial cells of Microcystis aeruginosa: part I: the key operating conditions. Sep. Purif. Technol. 52 (1), 84-94.

van de Wetering, P., Schuurmans-Nieuwenbroek, N.M.E., van Steenbergen, M.J., Crommelin, D.J.A., Hennink, W.E., 2000. Copolymers of 2-(dimethylamino)ethyl methacrylate with ethoxytriethylene glycol methacrylate or N-vinyl-pyrrolidone as gene transfer agents. J. Control. Release 64 (1-3), 193-203.

Yang, C., Dabros, T., Li, D., Czarnecki, J., Masliyah, J.H., 2001. Measurement of the zeta potential of gas bubbles in aqueous solutions by microelectrophoresis method. J. Colloid Interface Sci. 243 (1), 128-135.

Yoon, R.-H., Yordan, J.L., 1986. Zeta-potential measurements on microbubbles generated using various surfactants. J. Colloid Interface Sci. 113 (2), 430-438. 